Meta

Journal des traducteurs

Translators' Journal

\title{
Thème et texte
}

\section{Éveline Martin}

Volume 39, numéro 4, décembre 1994

Hommage à Bernard Quemada : termes et textes

URI : https://id.erudit.org/iderudit/003589ar

DOI : https://doi.org/10.7202/003589ar

Aller au sommaire du numéro

\section{Éditeur(s)}

Les Presses de l'Université de Montréal

\section{ISSN}

0026-0452 (imprimé)

1492-1421 (numérique)

Découvrir la revue

\section{Citer cet article}

Martin, É. (1994). Thème et texte. Meta, 39(4), 790-797.

https://doi.org/10.7202/003589ar

\section{Résumé de l'article}

Exposé de quelques problèmes qui se posent lors de l'indexation thématique d'un texte et de la recherche de contextes correspondant à un thème donné. Cet examen conduit à s'interroger sur la notion même de « thème " et à observer notamment le fonctionnement du mot thème aux $\mathrm{XIX}^{\mathrm{e}}$ et XX ${ }^{\mathrm{e}}$ siècles, dans le corpus de la base de données textuelles FRANTEXT : thèmes épisodiques, historiques, existentiels,... il s'agit toujours de pôles d'attraction plus ou moins spécifiques et susceptibles de déclencher une quelconque activité, souvent de réflexion ou de représentation, traduite fréquemment par un message oral ou écrit caractérisé lui-même par la récurrence des allusions aux dits thèmes. La compréhension du texte passe par le relevé des thèmes qui y sont traités, celle de chaque thème par l'étude des contextes qui l'illustrent.
Ce document est protégé par la loi sur le droit d'auteur. L'utilisation des services d'Érudit (y compris la reproduction) est assujettie à sa politique d'utilisation que vous pouvez consulter en ligne.

https://apropos.erudit.org/fr/usagers/politique-dutilisation/ 


\title{
THÈME ET TEXTE
}

ÉVELINE MARTIN

CNRS-INaLF, Paris, France

\begin{abstract}
Résumé
Exposé de quelques problèmes qui se posent lors de l'indexation thématique d'un texte et de la recherche de contextes correspondant à un thème donné. Cet examen conduit à $s$ 'interroger sur la notion même de "thème" et à observer notamment le fonctionnement du mot thème auX XIX ${ }^{e}$ et $X X^{e}$ siècles, dans le corpus de la base de données textuelles FRANTEXT: thèmes épisodiques, historiques, existentiels,... il s'agit toujours de pôles d'attraction plus ou moins spécifiques et susceptibles de déclencher une quelconque activité, souvent de réflexion ou de représentation, traduite fréquemment par un message oral ou écrit caractérisé lui-même par la récurrence des allusions aux dits thèmes. La compréhension du texte passe par le relevé des thèmes qui y sont traités, celle de chaque thème par l'étude des contextes qui l'illustrent.

Abstract

This article shows how a certain number of problems are posed when a text is indexed thematically and a theme is used to search for corresponding texts. Using the text base FRANTEXT, the study brings out the need for further thought on just what the idea of "theme" covers, and how the word thème is used in nineteenth and twentieth century texts: episodic, historic, existential themes..., always more or less specific poles of attraction capable of sparking off some activity, often of thought or representation, frequently translated by an oral or written message, characterized in turn by the recurrence of allusions to these themes. The understanding of the text means spotting the themes treated therein and the understanding of each theme entails the study of the contexts which illustrate it.
\end{abstract}

\section{PROBLÈMES DE THÉMATIQUE TEXTUELLE}

\section{Du texte au thème}

La forme d'analyse de texte la plus courante consiste à dégager les traits essentiels du contenu d'une œuvre, non pas les éléments du récit ou le déroulement de l'intrigue (si récit et intrigue il y a), mais bien les faits exemplaires, les idées dominantes, les pôles de réflexion récurrents, bref les thèmes abordés: il s'agit moins de résumer que d'attribuer une caractérisation thématique, d'indexer. Pour peu que l'opération ait pour principal objectif d'actionner un système de sélection automatique (quels textes illustrent le thème $d u$ double, par exemple), elle implique un langage de représentation des thèmes utilisé en amont (lors de l'intégration du texte) et/ou en aval (lors du questionnement). Contrairement à toute attente, l'indexation thématique d'un texte littéraire n'est pas bien différente de celle d'un texte dit «de référence», à la différence près que l'art de l'écrivain consiste le plus souvent à ne révéler que subrepticement les thèmes traités et que l'interprétation de l'analyste est alors dangereusement déterminante. On ne peut citer ici que l'un ou l'autre des problèmes qui se posent, et d'abord celui du niveau de généralisation auquel on décide de se situer: Les parents terribles illustre-t-il le thème du conflit de générations, de la famille, des relations parents / enfants? ... Avec Thérèse Desqueyroux a-t-on affaire au thème de la femme, du criminel - qui se trouve être une femme - du désespoir, du mariage, du mariage de convenance? ... Autant d'étiquettes qui, comme toutes celles qui sont citées ici, figurent bien dans les inventaires. Où s'arrêter? ... Et que faire des thèmes cachés? Si Genitrix illustre essentiellement le thème de la mère captative, il se trouve 
qu'à la fin du roman, peu avant sa mort, Félicité Cazenave devint tout autre et «son amour commença de ressembler à celui des autres mères, qui n'exige rien en échange de ce qu'il donne». En une courte formule, nous avons là le profil de la mère idéale que Mauriac fera vivre dans Le Mystère Frontenac sous les traits de Blanche dont Félicité est en quelque sorte le négatif. S. Rimmon-Kenan donne un exemple plus significatif emprunté à La mort d'Ivan Ilitch où, par le jeu des analepses, Tolstoï «traite du pouvoir de donner la vie qui appartient à la mort, à cause de la lucidité qu'elle aiguise» (Poétique, p. 405). C'est que le thème n'est pas toujours apparent dans le texte, du moins pas de façon récurrente.

Et d'ailleurs, à partir de quand un thème devient-il un thème? Peut-on parler du thème «du petit vélo à guidon chromé» chez Perec (Quel petit vélo à guidon chromé au fond de la cour?) ou de celui «du grand blond avec une chaussure noire» (le film), comme on parle du thème de «l'enfant au Moyen Age» ou de «l'ambition chez Balzac»? Quel taux de récurrence est-on en droit d'exiger pour qu'on puisse parler de thème? Et s'agira-t-il de récurrence interne (celle de tel thème dans telle œuvre particulière) ou d'une récurrence dans un ensemble plus ou moins délimité ?

L'indexation thématique d'un texte n'est donc pas une mince affaire: les descripteurs généraux sont faciles à manipuler mais mettent à plat l'information véhiculée par chaque texte, les descripteurs les plus spécifiques exigent une analyse détaillée du texte où une place doit être faite au peu explicite, voire à l'implicite. Resterait, pour bien faire, à user d'une syntaxe qui rendrait compte des relations entretenues par les thèmes dans l'œuvre considérée, soit par exemple pour Madame Bovary: mariage + province > ennui.

\section{Du thème au texte}

C'est la démarche inverse de la précédente. C'est la sélection - à partir d'une étiquette de thème donnée - de la documentation textuelle qui lui correspond.

L'indexation des textes du corpus fournit une première réponse, mais, dès que l'on souhaite accéder à des micro-contextes, on ne peut envisager d'indexation préalable, sinon des repérages par des critères de signifiants formels, dont on mesure bien la fragilité. Des expériences sont en cours sur le corpus de FRANTEXT qui consistent à constituer autour de chaque étiquette de thème un champ lexicologique non équivoque, fait de termes associés - mots simples et syntagmes - collectés dans les études et répertoires analogiques, et de vocables attestés avec une certaine fréquence, dans une collection représentative de contextes, de l'étiquette en question. La contrainte minimale imposée au moment de l'exploration d'un corpus de travail donné est la présence dans la phrase d'au moins deux vocables appartenant au champ lexicologique du thème concerné.

Les résultats obtenus sont pertinents dans leur grande majorité. On trouve par exemple des contextes comme ceux-ci, empruntés à des textes de genres différents, et destinés à servir de support à une étude sur l'expression de «l'espoir/l'espérance» dans des textes de 1950 à 1980 :

... d'un bout du monde à l'autre, au-dessus des champs de bataille comme à l'intérieur des usines, parmi les peuples opprimés aussi bien que chez les peuples libres, dans l'esprit des hommes de la rue comme dans celui des dirigeants, par-dessus les intérêts, les préjugés, les concurrences, s'élève et déferle aujourd'hui la vague des aspirations vers un idéal international. (De Gaulle, Mémoires de guerre: L'Appel, p. 677).

Ce sera, à l'occasion, une aspiration qu'il [le peintre] partage avec toute une collectivité, un idéal ou une foi. (R. Huyghe, Visible, 1955, p. 376).

Alors survint Jemappes. La confiance et l'enthousiasme s'exaltèrent sans mesure; les Montagnards ne furent pas moins ardents que les autres, et Robespierre, cette fois, n'essaya pas de résister au torrent. (G. Lefebvre, Révolution française, 1963, p. 294). 
En 1794, Condorcet écrivit son tableau des progrès de l'esprit humain, expression suprême de la pensée du XVIII ${ }^{\mathrm{e}}$ siècle, où, déjà proscrit, il attesta sa confiance héroïque dans les destinées de l'espèce humaine. (Id., Ibid., p. 592).

La pensée moléculaire est donc avant tout une attitude d'esprit, une sorte d'honnêteté et en même temps une sorte d'optimisme, une confiance dans les possibilités de la pensée. (A. David, Cybernétique, 1965, pp. 53-54).

Le cri d'un bébé dans la Corogne unit leurs yeux d'un regard ineffable : ils pensent ensemble à leur premier enfant. Un cri, un regard sont assez pour contenir la confiance, la promesse, l'union, l'avenir ... Comme l'étudiant a sa manière de présenter le firmament, la fille du pêcheur andalou présente à sa façon la Corogne ... (J.-P. Chabrol, Je t'aimerai, 1967, p. 152).

Olivier avait profondément ressentì l'enthousiasme d'une foule émerveillée de couleurs et de sons, respirant l'avenir à pleine poitrine, et, après que les lumières se furent éteintes, il en avait retenu des promesses prodigieuses, en dépit des forces maléfiques présentes au cour même des constructions comme cet aigle dominant le pavillon allemand et regardant ses proies futures. (R. Sabatier, Fillettes chantantes, 1980, pp. 111-112).

Qu'il s'agisse de dégager le ou les thèmes d'un texte donné en vue de le caractériser, de le résumer ou de l'indexer, qu'il s'agisse de repérer, de reconnaitre un thème donné dans un corpus plus ou moins grand, il convient avant tout de savoir ce qui peut légitimement être appelé thème, ce que recouvre couramment ce mot: l'examen des contextes est révélateur d'une extrême diversité des conditions d'emploi du mot, en même temps que d'une certaine complexité de la notion, indépendamment même de sa définition canonique.

\section{THÈME EN CONTEXTE}

Un examen du fonctionnement du mot thème aux $\mathrm{XIX}^{\mathrm{e}}$ et $\mathrm{XX}^{\mathrm{e}}$ siècles dans le corpus de FRANTEXT ${ }^{1}$ montre qu'il est des thèmes favoris, de prédilection, préférés, à la mode, fréquents, en vogue, classiques; un thème est cher à tel ou tel; il est des thèmes uniques, populaires, traditionnels, anciens, originels, habituels, constants, permanents, éternels, intemporels, inépuisables et inépuisés; il est des thèmes usés, mais aussi des thèmes renouvelés; il est de beaux thèmes ..., des thèmes admirables, pittoresques, majestueux ..., de grands thèmes, des thèmes immenses, abondants, vastes, mais aussi des thèmes insignifiants, communs, ordinaires ou bizarres, moraux ou immoraux, voire grossiers; des thèmes simples et simplistes, intelligibles, expressifs, concrets, pratiques ou vagues, complexes, abstraits, mythiques; il est de doctes thèmes ..., des thèmes drastiques; il est des thèmes amoureux, romantiques, poétiques, sentimentaux, idéalistes ..., des thèmes matérialistes, des thèmes commerciaux, économiques, physiologiques, philosophiques et métaphysiques, des thèmes humains, de consolation, des thèmes sociaux, socialistes, collectivistes ..., politiques, religieux, chrétiens, théologiques, sacerdotaux; un thème peut être général, central, majeur, fondamental, essentiel, dominant, directeur ou directif, générateur, conducteur, inspirateur; il est des thèmes d'orientation, de ralliement, de propagande, d'imitation; des thèmes de revendications, de préoccupations, de recherches, de travail, d'études, de manouvre ..., des thèmes d'imagination, de rêveries, de pensées, des thèmes delà réflexions, des thèmes à méditation, des thèmes de paroles, entretiens, conversations, causeries, déclamations, développements rhétoriques, sermons, des thèmes d'articles, de lettres, de narrations, de romans, de légendes; on organise une exposition, on réalise une émission sur le thème de ... On peut avoir un thème de vie ... On choisit un thème, on le fournit, on le donne, on l'annonce, on l'aborde, on le présente, on le propose, on l'esquisse, on le met en cuvre, on l'étudie, on le creuse, on l'illustre, on le déroule, on le crée, on le réalise, on l'étudie, on le raconte, on le ressasse, on le reprend, l'enrichit, le transforme; on varie les thèmes; on est jeté, lancé sur un thème, on fonce 
dans un thème; on parle, on écrit, on médite, on moralise, on improvise, on brode, on joue, on s'exerce, on divague sur un thème; on se prépare à un thème, on s'écarte d'un thème, on prend quelque chose pour thème, on s'appuie sur un thème, on puise dans des thèmes; la pensée gravite, on brode autour de thèmes; quelque chose ou quelqu'un $a$ pour thème, fait le thème de quelqu'un ou quelque chose ..., on pense par thèmes, on a le choix des thèmes; un thème a des aspects, des facettes; il peut donner lieu à des développements, des fantaisies, des modulations, des variations (même en dehors du contexte musical); les thèmes peuvent changer, reparaître, revenir, se rejoindre ...

Le même corpus textuel, celui des contextes de thème(s), fournit un catalogue intéressant de ce qui est considéré comme thème. On trouve majoritairement ce qu'on pourrait appeler des thèmes épisodiques. Un fait, un trait ponctuel, «local», va, investi de l'importance qui lui est donnée dans le contexte où il est cité, faire l'objet de commentaires appuyés: le projet d'une décoration de restaurant laide et couteuse (Goncourt, Journal, p. 960), l' unique plaidoirie d'un avocat médiocre (Theuriet, Maison, p. 7), la maladresse d'un palefrenier et la semonce qui s'ensuit (Gobineau, Pléiades, p. 31), la naissance d'un enfant adultérin (Zola, M. Férat, p. 51), la ferme d'un avoué dans la voiture d'une comtesse (Champfleury, Bourgeois, p. 104), une expropriation (Duhamel, Notaire, p. 167), l'omnibus à prendre, le dernier dîner, le dernier sermon, la dernière robe, le dernier air de piano joué. Le thème peut être hypothétique (Si les eaux se retiraient, le Duncan, avant trois jours, reverrait les voyageurs à son bord, J. Verne, Enfants, p. 221); il peut n'être qu'un rêve (Si j’étais né prince souverain ..., Vogüé, Morts, p. 48).

Mais peuvent aussi devenir thèmes des événements historiques, politiques, donc d'un intérêt plus général : la candidature de Deschanel (Barrès, Cahiers, p. 249), le contrôle parlementaire (Ibid., p. 317), l'embranchement de Niort à Angers (Zola, Son Excellence, p. 262), la fragilité du gouvernement de Louis-Philippe (Stendhal, L. Leuwen, p. 162), la trahison dont la France est victime au Levant (De Gaulle, Mémoires, Appel, p. 408), les guerres de religion (Bainville, Hist. de Fr., p. 163), la découverte de la France (Vercors, Silence, p. 49).

Parfois, plus généralement encore, c'est de la condition des humains qu'il s'agit: la malédiction des faibles (Gautier, C. Fracasse, p. 155), la passion des femmes dans le mariage (Goncourt, Ch. Demailly, p. 252), la duplicité féminine et les intermittences du caur (Béguin, Ame, p. 715), le petit orphelin qui n' a ni père ni mère (Sabatier, Noisettes, p. 135), la défense des frontières (Service militaire, p. 50), la sève montante, le bâton qui reverdit (Menon-Lecotte, Village, p. 110), la vie du mineur (Schneider, Charbon, p. 227), l'opposition entre la vie qu'on subit et la vie qu'on rêve (Guéhenno, Jean-Jacques, p. 33), la brièveté de la vie (Du Bos, Journal II, p. 38), le jeune héros qui se retrouve dans la lumière de l'aube, fort de sa victoire sur les spectres nocturnes (Green, Journal I, p. 223).

On notera que les intitulés comportant un agrégat, une combinaison de thèmes ( $l$ 'angoisse de la liberté [Riccur, Volonté, p. 63]; la joie dans l'obéissance [Jouve, Paulina, p. 175]) suggèrent une prise de position du locuteur, auteur ou personnage, et nombre de désignations un peu plus détaillées de thèmes impliquent une réflexion préalable, un choix, et incluent la sentence: le désir d'un meilleur état est la source de tout le mal dans le monde (Renan, Avenir, p. 19); qui attaque constamment le travail national? la presse (Reybaud, J. Paturot, p. 303); le langage n'exprime qu'une partie de la réalité (Benda, France, p. 50); la ligne de démarcation entre la gauche et la droite passe toujours par la laïcité (Philosophie. Religion, dir. G. Berger, p. 4613); la science sociale est possible (Vedel, Droit, p. 15), l'histoire est résurrection du passé (Gurvitch, Sociologie, p. 85); un jeune inconnu révèle sa noble origine rien qu'à la façon dont il dépèce une bête abattue (Faral, St Louis, p. 34). Il faut bien noter que les jugements ne sont que cités par les auteurs, ceux-ci sont bien loin de les prendre toujours à leur compte. 
On trouve chez J. Lemaitre (1939) un court inventaire des thèmes de son temps: l'aspiration de nos âmes vers l'Infini, l'écrasement de l' homme éphémère et borné sous l'immensité et l'éternité de l' univers, l'angoisse du doute, la communion de l'âme avec la nature (Les Contemporains, p. 38), l'âcreté des amours coupables et hâtives dans les bouges ou les fiacres errants; la solitude des âmes qui ne peuvent s'unir, et la vanité des caresses, qui ne joignent que les corps; la solitude libératrice de la vieillesse, qui affranchit de la femme et qui achève en nous la bonté; le désir de s'éteindre en écoutant un chant de nourrice (Ibid., p. 55). On en trouve d'autres chez F. Ambrière (1946): l'Europe nouvelle; le travail de guerre, devoir sacré; la corruption française et la régénération par la pénitence nationale, l'urgence d'adorer le plus fort et de se mettre à son école (Grandes vacances, p. 135).

À côté des étiquettes traditionnelles dont on a donné des exemples attestés, on trouve des désignations plus particulières dont certaines sont propres à un auteur ou à un domaine (les cerveaux géants [Ruyer, Cybernétique, p. 85]; la hutte de l'ermite [Bachelard, Espace, p. 46]); d'autres se distinguent par la typographie (En fait, les petits morveux devaient être capables de nous tirer des larmes en tournant une pub pour un groupement d'assurances sur le thème: BÂTISSONS LEUR AVENIR [Djian, Matin, p. 309]), la langue (natürlicher Weltbegriff [conception du monde] de Husserl cité par Merleau-Ponty [Phénoménologie, p. 1]). D'autres constituent un véritable énoncé qui est censé être dans l'esprit du locuteur toutes les fois qu'il développe son thème sous une forme ou l'autre (Celui qui te parle n'est pas n'importe qui [Chabrol, Je t'aimerai, p. 218]; Pourquoi $m^{\prime}$ obliges-tu, c'est peut-être nécessaire, mais tellement au-dessus de mes forces? [Monesi, Nature morte, p. 214]).

Ces exemples, exemples d'auteurs en quelque sorte, ont seulement valeur de témoignage; ils sont la preuve qu'au moins une fois et à un moment donné, les concepts cités ont été considérés comme des thèmes. Un relevé exhaustif, qui n'aurait pas sa place ici, révélerait encore leur extrême diversité, du thème de l'apéro, qui n'est rien d'autre que la répétition lancinante du «que puis-je vous offrir?» (Blier, Valseuses, p. 249) à celui des services des PTT, hier, aujourd' hui et demain (titre d'une exposition citée dans Administration des P. et T., p. 40) en passant par les jardins de Paris (Dorival, Peintre du $20^{e}$ s., p. 66), ou l' unification des terminologies (Jocard, Tourisme, p. 89). Aphorismes, truismes, clichés parfois, il s'agit de thèmes qu'on pourrait qualifier de «propositionnels». Il en est d'une autre nature, plus «conceptuelle»; ils forment une véritable nomenclature de conceptsclés: l'alcoolisme (Cacérès, Hist. Éducation, p. 115), l'amitié (Blanche, Modèles, p. 122), l'amour impossible (Mounier, Caractère, p. 152), l'animisme (Bergson, Deux sources, p. 197), le bal (Anouilh, Répétition, p. 19), la beauté (Maurois, Climats, p. 19), la culture (Hist. science, p. 1523), la dignité humaine (Sartre, Existentialisme, p. 107), la disparition (Poulet, Métamorphoses, p. 180), la douleur (Djian, Matin, p. 116), l'éducation des enfants (Gide, Si le grain, p. 482), l'égoüsme (Valéry, Variété $I I$, p. 88), l'enfance (Sagan, Chamade, p. 14), l'enseignement supérieur (Antoine-Passeron, Réforme université, p. 113), l'évasion (Maurois, Op. cit., p. 207), l'évolution (Hist. gén. des sciences III, p. 549), la fête (Anouilh, Op. cit., p. 19), la gloire de Dieu (Gilson, Esprit, p. 169), l'inceste (Artaud, Théâtre et son double, p. 105), l'irrationnel (Camus, Mythe, p. 62), la jalousie (Blanche, Op. cit., p. 122), le loisir (Dumazedier-Ripert, Loisir, p. 27), la mer (Billy, Intrö̈bo, p. 184), la magie (Bergson, Op. cit., p. 197), la modestie (Blanche, Op. cit., p. 122), le néant (Saint-John Perse, Exil, p. 212), la paix (Meynaud, Groupes de pression, p. 64), la personnalité humaine (Amadou, Parapsychologie, p. 34), la productivité (Chenot, Entreprises, p. 35), le progrès (Benda, Trahison, p. 244), la province natale (Durry M.-J., Nerval, p. 14), les ruines (Febvre, Combats, p. 35), la sérénité (Maurois, Ibid., p. 277), le silence (Sabatier, Allumettes, p. 178), la solitude (Lacroix, Marxisme, p. 77), le soupçon (Blanche, Op. cit., 
p. 122), les vertus (Fulcanelli, Demeures, p. 192), la ville (Lesourd-Gérard, Hist. économique, p. 258).

Si l'on cherche à présent les contextes de thème dans le répertoire lexicologique $\mathrm{BORNEO}^{2}$, on voit apparaitre des syntagmes comme

- thème mémorisable

Voilà une super mayo entre l'image et le son, bien mixée, bien enregistrée, et même s'il n'y a pas de thème extraordinairement mémorisable, il y a le climat, hyper réussi. (F. Tressour, Première, mars 1985, p. 127),

thème rétro

C'est ce qui rend si nostalgique l'appel d'Alexandre Sanguinetti à une «nouvelle résistance» et fait un thème "rétro" de ce qu'il sent comme ardente obligation d'avenir. (J. Ozouf, Le Nouvel Observateur, 7 juin 1976, p. 37),

- thème sécuritaire

Les mêmes pressions ont fait du supplément économique le must de tous les journaux et magazines dans la presse écrite. Revenue au pouvoir, la droite tente vainement de contrebalancer cette dictature des indices économiques en brandissant le thème sécuritaire. (F. de Closets, Le Nouvel Observateur, 11 sept. 1986, p. 54),

- thème ultra-rebattu

Pour faire bon poids, on a même ajouté [dans le film «Natty Gann»] un autre thème ultrarebattu, celui de l'amitié d'un enfant et d'un animal, en l'occurrence un chien-loup pourri de talent. (M.-F. Leclère, Le Point, 3 févr. 1986, p. 13)

et, par analogie plaisante de forme, thème gastrologique (titre d'un commentaire d'information de L'Express du 19 déc. 85, p. 113).

Thème figure en composition dans :

thème-parc

En France, dans le froissement des annonces, trois projets émergent. Schtroumpfland, en Lorraine, est le plus avancé. Ce «thème-parc» dressé sur les anciens laminoirs d'Hagondange devrait recevoir quatre millions de visiteurs à partir de 1988. (Le Point, 18 mars 1985, p. 92),

- thème-phare

C'est à ce moment de l'histoire qu'entrent en scène l'Union de la Gauche et, avec elle, de nouveaux protagonistes du drame de la Manu. Thème-phare de la liste P.C.-P.S., pendant la campagne municipale: «Pas de licenciements ni de démantèlement à Manufrance.» (F.-O. Giesbert, Le Nouvel Observateur, 26 févr. 1979, p. 96),

thème-prétexte

Cette année, il [le Festival de La Chaise-Dieu] met dos à dos la France et l'Allemagne. On ne sait pas qui va gagner. Mais ce thème-prétexte permettra d'entendre face au «Requiem» de Mozart [...]. (J. Drillon, Le Nouvel Observateur, 16 août 1985, p. 15).

\section{On évoque la radio à thème}

Je me suis peu à peu rendu compte que l'avenir résidait dans la radio «thématique» permettant à l'auditeur de faire son choix en fonction des spécialités proposées. La radio à thème c'est l'assurance, pour l'auditeur, d'une plus grande liberté, pour les émissions, d'une plus grande qualité. (R. Faure, Le Figaro magazine, 10 oct. 1987, p. 85),

des croisières à thème

... pour les happy few, des croisières à thème: musique, peinture, gastronomie, bridge, échecs, cinéma. (Le Point, 26 avr. 1986, p. 91), 
les restaurants à thème

Enfin, troisième voie d'《avenir», les restaurants «à thème» organisés en chaîne sous une enseigne commune, tels les hôtels. [...] le poisson (la Criée), le pain (les pizzerias), le pain et le vin (les wine-bars). Le thème peut être aussi un décor: les brasseries Flo. Ou une clientèle: les femmes à l'Amanguier. Ou le dépaysement: dans les Bistros de la Gare et les Bistrots romains. Ou la musique au City Rock Café. (P.-M. Doutrelant, Le Nouvel Observateur, 16 août 1985 , p. 38),

\section{le tourisme à thème}

Autre fait nouveau, les Français sont de plus en plus nombreux à choisir sur catalogue des voyages en France. Cela s'explique, d'une part par l'organisation de plus en plus fréquente des séjours à forfait de sports d'hiver, et de l'autre, par la vogue croissante des Antilles et enfin, à un moindre degré, par le développement du tourisme à thème - poterie ou vélo dans les verts pâturages de l'Hexagone. (C. Bergeron, Le Point, 13 juin 1977, p. 90).

En ce qui concerne les «référents» de thème, il s'agit de choses aussi différentes que

- «le chacun pour soi»

... sous des dehors modernistes, le thème «coluchien» du «chacun pour soi» est en réalité singulièrement égoïste, démoralisateur et poujadiste, comme sont poujadistes les foules dites populaires, mais payant cher leur billet, qui se précipitent au théâtre du Gymnase. (A. Chambraud, Le Point, 24 nov. 1980, p. 44),

- «le labyrinthe»

... le premier film «riche» de Raul Ruiz, «Le Territoire», qui reprend le thème borgésien du labyrinthe pour le corser de traits d'humour surréalistes. (M. Mardore, Le Nouvel Observateur, 17 oct. 1981, p. 124),

ou ... «la chasse aux chevaux sauvages par des marginaux à bout de souffle»

LES DÉSAXÉS (1960) de John Huston [...] Dernier chant de trois monstres sacrés, sur un thème (d'Arthur Miller) ultra-signifiant: la chasse aux chevaux sauvages par des marginaux à bout de souffle... (M. Mardore, Le Nouvel Observateur, 26 avr. 1985, p. 22).

On notera au passage les thèmes borgésien et coluchien auxquels on peut ajouter

\section{- le thème failevicien}

Qui soigne les ceps, qui taillera les treilles, sans être sûr d'en récolter les grappes? C'est le thème, terriblement failevicien, de ce téléfilm [«L'Héritage» de Maurice Failevic]... (P. Aubert, L'Express, 24 juin 1983, p. 20),

- et le thème mizoguchien

LA FÊTE DE GION (1953) de Kenji Mizoguchi [...] Deux femmes, deux geishas, préservent leur affection et, surtout pour l'aînée envers la débutante, leur dévouement, dans un monde hostile, cynique, où elles ne peuvent être qu'exploitées... Ce thème, ô combien mizoguchien, n'a pas fait de ce film un classique. (F. Vitoux, Le Nouvel Observateur, 11 mai 1984, p. 18).

On note encore dans la même base l'emploi d'un verbe thémer, probablement hapax:

Éventuellement, un nom peut pousser l'audace jusqu'à remplacer un verbe. Exemples: «Je thème six fois par an (pub pour un «bricolage-magazine», Création, op. cit. [novembre 1986]). «Je doudoune en $48 \mathrm{~h}$ chrono». (campagne La Redoute)... (L. Mercadet, N. Roiret, Actuel, nov. 1987, p. 80).

Qu'ont en commun ces thèmes nombreux - cités comme tels dans les textes et fort différents les uns des autres - («l'histoire est résurrection du passé» ou l'amitié d'un 
enfant et d'un animal), les poncifs (le gouffre, la fête) de l'analyste qui les traque (quels thèmes dans ...?) ou de l'anthologiste qui en fait des motifs (le cheval dans la littérature)? Avant tout peut-être le fait qu'il s'agit toujours d'un certain pôle d'attraction plus ou moins spécifique et susceptible de déclencher une quelconque activité, souvent de réflexion ou de représentation, traduite fréquemment par un message oral ou écrit caractérisé lui-même par la récurrence des allusions au dit thème. Le relevé méthodique de ces pôles d'attraction constitue la première étape de la compréhension d'un texte. De même que, réciproquement, la collecte de contextes illustrant un même thème constitue la première étape de compréhension de ce thème. Ceci suffit à faire du thème un élément déterminant du texte, et du texte le moyen d'expression privilégié du thème.

\section{Notes}

1. Pour ce qui concerne FRANTEXT, consulter le Répertoire des publications et produits informatisés de I'INaLF, 1993, pp. 31-32 (les références des extraits de textes correspondent aux éditions du corpus de cette base de données).

2. Base d'Observation et de Recensement des Néologismes (relevés dans la presse), alimentée par l'Équipe de Néologie de l'INaLF. 